International Mathematical Forum, Vol. 6, 2011, no. 43, 2117 - 2124

\title{
On the Global Asymptotic Stability of a Fourth-Order Rational Difference Equation
}

\author{
Vu Van Khuong \\ Department of Mathematics \\ Hung Yen University of Technology and Education \\ Khoai Chau District, Hung Yen, Viet Nam \\ vuvankhuong@gmail.com \\ Le Hong Lan \\ Department of Mathematical Analysis \\ University of Transport and Communications \\ Hanoi City, Viet Nam \\ honglanle229@gmail.com
}

\begin{abstract}
In this work, we investigate the global stability of the following fourth-order rational difference equation

$$
x_{n+1}=\frac{x_{n}^{b} x_{n-1} x_{n-3}^{b}+x_{n}^{b}+x_{n-1}+x_{n-3}^{b}+a}{x_{n}^{b} x_{n-1}+x_{n-1} x_{n-3}^{b}+x_{n}^{b} x_{n-3}^{b}+1+a}, n=0,1,2, \ldots
$$

where $a, b \in[0, \infty)$ and the initial values $x_{-3}, x_{-2}, x_{-1}, x_{0} \in(0, \infty)$.

Mathematics Subject Classification: 39A10
\end{abstract}

Keywords: Rational difference equation, global stability

\section{Introduction}

Rational difference equations can be look very simple, but in fact their global behaviors are mostly very complicated.

$\mathrm{Li}$ and Zhu [1] are obtained a sufficient condition to quarante the global asymptotic stability of the following recursive sequence

$$
x_{n+1}=\frac{x_{n} x_{n-1}^{b}+x_{n-2}^{b}+a}{x_{n-1}^{b}+x_{n} x_{n-2}^{b}+a}, n=0,1,2, \ldots
$$


where $a, b \in[0, \infty)$ and the initial values $x_{-2}, x_{-1}, x_{0} \in(0, \infty)$.

$\mathrm{Li}[2]$ use a new method to investigate the qualitative properties of the following rational difference equation

$$
x_{n+1}=\frac{x_{n} x_{n-1} x_{n-3}+x_{n}+x_{n-1}+x_{n-3}+a}{x_{n} x_{n-1}+x_{n} x_{n-3}+x_{n-1} x_{n-3}+1+a}, \quad n=0,1,2, \ldots
$$

V. V. Khuong [3, 4] investigated the global behavior of the following fourthorder rational difference equation

$$
x_{n+1}=\frac{x_{n} x_{n-1}^{b} x_{n-3}+x_{n}+x_{n-1}^{b}+x_{n-3}+a}{x_{n} x_{n-1}^{b}+x_{n-1}^{b} x_{n-3}+x_{n} x_{n-3}+1+a}, n=0,1,2, \ldots
$$

To be motivated by the above studies, in this paper, we consider the following nonlinear difference equation

$$
x_{n+1}=\frac{x_{n}^{b} x_{n-1} x_{n-3}^{b}+x_{n}^{b}+x_{n-1}+x_{n-3}^{b}+a}{x_{n}^{b} x_{n-1}+x_{n-1} x_{n-3}^{b}+x_{n}^{b} x_{n-3}^{b}+1+a}, n=0,1,2, \ldots
$$

where $a, b \in[0, \infty)$ and the initial values $x_{-3}, x_{-2}, x_{-1}, x_{0} \in(0, \infty)$.

We review some results which will be useful in our investigation.

Definition 1.1. Let $I \subset \mathbb{R}$ and $f: I^{k+1} \rightarrow I$ be a continuously differentiable function. Then for every set of initial conditions $x_{-k}, x_{-k+1}, \ldots, x_{0} \in I$, the difference equation

$$
x_{n+1}=f\left(x_{n}, x_{n-1}, \ldots, x_{n-k}\right), \quad n=0,1,2, \ldots
$$

has an unique solution $\left\{x_{n}\right\}_{n=-k}^{\infty}$.

A point $\bar{x} \in I$ is called an equilibrium point of equation (5) if $\bar{x}=f(\bar{x}, \bar{x}, \ldots, \bar{x})$.

Definition 1.2. Let $\bar{x}$ be the equilibrium point of the Eq. (5).

(i) The equilibrium point $\bar{x}$ of Eq. (5) is called locally stable if for every $\epsilon>0$, there exists $\delta>0$ such that for all $x_{-k}, x_{-k+1}, \ldots, x_{0} \in I$, with

$$
\left|x_{-k}-\bar{x}\right|+\left|x_{-k+1}-\bar{x}\right|+\ldots+\left|x_{0}-\bar{x}\right|<\delta,
$$

we have

$$
\left|x_{n}-\bar{x}\right|<\epsilon \text { for all } n \geq-k
$$

(ii) The equilibrium point $\bar{x}$ of Eq. (6) is called a global attractor if for every $x_{-k}, x_{-k+1}, \ldots, x_{0} \in I$, we have

$$
\lim _{n \rightarrow \infty} x_{n}=\bar{x}
$$


(iii) The equilibrium point $\bar{x}$ of Eq. (5) is called a global asymptotically stable if it is locally stable and a global attractor.

Definition 1.3. A positive semicycle of a solution $\left\{x_{n}\right\}_{n=-3}^{\infty}$ consists of a "string" of terms $\left\{x_{l}, x_{l+1}, \ldots, x_{m}\right\}$, all greater than or equal to the equilibrium $\bar{x}$, with $l \geq-3$ and $m \leq \infty$ and such that

$$
\text { either } l=-3 \text {, or } l>-3 \text { and } x_{l-1}<\bar{x} \text {. }
$$

and

$$
\text { either } m=\infty \text {, or } m<\infty \text { and } x_{m+1}<\bar{x} \text {. }
$$

A negative semicycle of a solution $\left(x_{n}\right)$ consists of a "string" of terms $\left\{x_{l}, x_{l+1}, \ldots, x_{m}\right\}$, all less than to $\bar{x}$, with $l \geq-3$ and $m \leq \infty$ and such that

$$
\text { either } l=-3 \text { or } l>-3 \text { and } x_{l-1} \geq \bar{x} \text {. }
$$

and

$$
\text { either } m=\infty \text { or } m<\infty \text { and } x_{m+1} \geq \bar{x} \text {. }
$$

The length of a semicycle is the number of the total terms contained in it.

Definition 1.4. A solution $\left\{x_{n}\right\}_{n=-3}^{\infty}$ of Eq. (5) is said to be enventually trivial if $x_{n}$ eventually equal to $\bar{x}=1$; otherwise, the solution is said to be nontrivial.

\section{Several Lemmas}

It is easy to see that the positive equilibrium point $\bar{x}$ of Eq. (1) satisfies

$$
\bar{x}=\frac{\bar{x}^{1+2 b}+\bar{x}+2 \bar{x}^{b}+a}{2 \bar{x}^{1+b}+\bar{x}^{2 b}+1+a}
$$

from which one can see that Eq. (1) has an unique positive equilibrium $\bar{x}=1$.

Lemma 2.1. A positive solution $\left\{x_{n}\right\}_{n=-k}^{\infty}$ of Eq. (1) is eventually equal to 1 if and only if

$$
\left(x_{-3}-1\right)\left(x_{-1}-1\right)\left(x_{0}-1\right)\left(x_{1}-1\right)=0
$$

Proof. Assume the (9) holds. Then according to Eq. (1), it is easy to see that $x_{n}=1$ for $n \geq 1$.

Conversely, assume that

$$
\left(x_{-3}-1\right)\left(x_{-1}-1\right)\left(x_{0}-1\right)\left(x_{1}-1\right) \neq 0
$$


Then one can show that $x_{n} \neq 1$ for any $n \geq 1$.

Assume the contrary that for some $N \geq 1$,

$$
x_{N}=1 \text { and that } x_{n} \neq 1 \text { for }-3 \leq n \leq N-1
$$

It is easy to see that

$$
1=x_{N}=\frac{x_{N-1}^{b} x_{N-2} x_{N-4}^{b}+x_{N-1}^{b}+x_{N-2}+x_{N-4}^{b}+a}{x_{N-1}^{b} x_{N-2}+x_{N-2} x_{N-4}^{b}+x_{N-1}^{b} x_{N-4}^{b}+1+a},
$$

which implies $\left(x_{N-4}^{b}-1\right)\left(x_{N-2}^{b}-1\right)\left(x_{N-1}-1\right)=0$. Obviously, this contradicts (11).

Remark 2.1. If the initial conditions do not satisfy Eq. (9), then, for any solution $\left\{x_{n}\right\}$ of Eq. (1), $x_{n} \neq 1$ for $n \geq-3$. Here, the solution is a nontrivial one.

Lemma 2.2. Let $\left\{x_{n}\right\}_{n=-3}^{\infty}$ be a nontrivial positive solution of Eq. (1). Then the following conclusions are true for $n \geq 0$.

(a) $\left(x_{n+1}-1\right)\left(x_{n}-1\right)\left(x_{n-1}-1\right)\left(x_{n-3}-1\right)>0$

(b) $\left(x_{n+1}-x_{n-1}\right)\left(x_{n-1}-1\right)<0$.

(c) $\left(x_{n+1}-x_{n}^{b}\right)\left(x_{n}^{b}-1\right)<0$.

(d) $\left(x_{n+1}-x_{n-3}^{b}\right)\left(x_{n-3}^{b}-1\right)<0$.

Proof. It follows in light of Eq. (5) that

$$
x_{n+1}-1=\frac{\left(x_{n}^{b}-1\right)\left(x_{n-1}-1\right)\left(x_{n-3}^{b}-1\right)}{x_{n}^{b} x_{n-1}+x_{n-1} x_{n-3}^{b}+x_{n}^{b} x_{n-3}^{b}+1+a}, \quad n=0,1,2, \ldots
$$

and

$$
x_{n+1}-x_{n}^{b}=\frac{\left(1-x_{n}^{b}\right)\left[x_{n}^{b}\left(1+x_{n-1}\right)+x_{n-3}^{b}+x_{n-3}^{b} x_{n-1}+a\right]}{x_{n}^{b} x_{n-1}+x_{n-1} x_{n-3}^{b}+x_{n}^{b} x_{n-3}^{b}+1+a}, \quad n=0,1,2, \ldots
$$

and

$x_{n+1}-x_{n-1}=\frac{\left(1-x_{n-1}\right)\left[x_{n}^{b}\left(1+x_{n-1}\right)+x_{n-3}^{b}+x_{n-3}^{b} x_{n-1}+a\right]}{x_{n}^{b} x_{n-1}+x_{n-1} x_{n-3}^{b}+x_{n}^{b} x_{n-3}^{b}+1+a}, \quad n=0,1,2, \ldots$

and

$$
x_{n+1}-x_{n-3}^{b}=\frac{\left(1-x_{n-3}^{b}\right)\left[x_{n-1}\left(1+x_{n-3}^{b}\right)+x_{n-3}^{b}\left(1+x_{n-1}\right)+a\right]}{x_{n}^{b} x_{n-1}+x_{n-1} x_{n-3}^{b}+x_{n}^{b} x_{n-3}^{b}+1+a}, n=0,1,2, \ldots
$$


Lemma 2.3. If $x_{-3}<x_{-2}<x_{-1}<x_{0}<1$, then $\left\{x_{n}\right\}_{n=-3}^{\infty}$ has a negative semicycle with an infinite number of terms and it monotonically tends to the positive equilibrium point $\bar{x}=1$.

Proof. If $x_{-3}<x_{-2}<x_{-1}<x_{0}<1$ then from Lemma $2.2 \mathrm{~b}$ ), for $n \geq-3$ we have

$$
\begin{gathered}
x_{0}<x_{2}<x_{4}<\ldots<x_{2 n}<\ldots<1 \text { and } \\
x_{1}<x_{3}<x_{5}<\ldots<x_{2 n+1}<\ldots<1 .
\end{gathered}
$$

So, the limits

$$
\lim _{n \rightarrow \infty} x_{2 n}=L \text { and } \lim _{n \rightarrow \infty} x_{2 n+1}=M
$$

exist and finite.

Taking the limit on both sides of Eq. (1), we get

$$
L=\frac{M^{b} L L^{b}+M^{b}+L+L^{b}+a}{M^{b} L+L^{1+b}+M^{b} L^{b}+1+a}=1 \Longrightarrow L=1
$$

and

$$
M=\frac{L^{b} M^{1+b}+L^{b}+M+M^{b}+a}{L^{b} M+M^{1+b}+M^{b} L^{b}+1+a}=1 \Longrightarrow M=1
$$

We can easily se that $\left\{x_{n}\right\}_{n=-3}^{\infty}$ tends to the positive equilibrium point $\bar{x}=$ 1.

\section{$3 \quad$ Main results}

First we analyze the structure of the semicycles of nontrivial solutions of Eq. (1). Here we confine us to consider the situation of the strictly oscillatory solution of Eq. (1). From [6, 7], we have the following theorem.

Theorem 3.1. Let $\left\{x_{n}\right\}_{n=-3}^{\infty}$ be a strictly oscillatory solution of Eq. (1). Then the rule for the lengths of positive and negative semicycles of this solution to successively occur is

$$
\begin{aligned}
& \ldots, 3^{+}, 2^{-}, 1^{+}, 1^{-}, 3^{+}, 2^{-}, 1^{+}, 1^{-}, \ldots \\
& \text { or } \ldots, 3^{-}, 2^{+}, 1^{-}, 1^{+}, 3^{-}, 2^{+}, 1^{-}, 1^{+}, \ldots
\end{aligned}
$$

Theorem 3.2. Assume that $a, b \in[0, \infty), b>0$. Then the positive equilibrium of Eq. (1) is globally asymptotically stable.

Proof. We must prove that the positive equilibrium point $\bar{x}$ of Eq. (1) is both locally asymptotically stable and globally attractor. The linearized equation of Eq. (1) about the positive equilibrium $\bar{x}=1$ is

$$
y_{n+1}=0 . y_{n}+0 . y_{n-1}+0 . y_{n-2}+0 . y_{n-3}, \quad n=0,1,2, \ldots
$$


By virtue of ([1], Remark 1.3.7), $\bar{x}$ is locally asymptotically stable. It remains to verify that every positive solution $\left\{x_{n}\right\}_{n=-3}^{\infty}$ of Eq. (1) converges to $\bar{x}=1$ as $n \rightarrow \infty$. Namely, we want to prove

$$
\lim _{n \rightarrow \infty} x_{n}=\bar{x}=1
$$

If the solution is nonoscillatory about the positive equilibrium point $\bar{x}$ of Eq. (1), then we know from Lemma 2.1 and Lemma 2.2, the solution is either equal to 1 or eventually negative one which has an infinite number of terms and monotonically tends to the positive equilibrium point $\bar{x}$ of Eq. (1), and so Eq. (15) holds. Therefore, it sufficient to prove that Eq. (15) holds for the solution to be strictly oscillatory.

Consider now $\left\{x_{n}\right\}_{n=-3}^{\infty}$ to be strictly oscillatory about the possitive equilibrium point $\bar{x}$ of Eq. (1). By virtue of Thoerem 3.1, the solution of Eq. (1) has the positive and negative semicycles of the form

$\ldots, 3^{+}, 2^{-}, 1^{+}, 1^{-}, 3^{+}, 2^{-}, 1^{+}, 1^{-}, \ldots$ or $\ldots, 3^{-}, 2^{+}, 1^{-}, 1^{+}, 3^{-}, 2^{+}, 1^{-}, 1^{+}, \ldots$

First, we investigate the case where the rule for the lengths of positive and negative semicycles which occur successively is

$$
\ldots, 3^{+}, 2^{-}, 1^{+}, 1^{-}, 3^{+}, 2^{-}, 1^{+}, 1^{-}, \ldots
$$

It can be periodically expressed as follows:

$$
\left\{x_{p+7 n}, x_{p+7 n+1}, x_{p+7 n+2}\right\}^{+},\left\{x_{p+7 n+3}, x_{p+7 n+4}\right\}^{-},\left\{x_{p+7 n+5}\right\}^{+},\left\{x_{p+7 n+6}\right\}^{-}, \ldots
$$

$n=0,1,2, \ldots$

From Lemma $2.2 \mathrm{~b}$ ) the following results can be easily observed

(i) $x_{p+7 n+2}<x_{p+7 n}$;

(ii) $x_{p+7 n+6}>x_{p+7 n+4}$.

One can easily prove the following inequalities

$$
\begin{aligned}
& \text { (iii) } \frac{1}{x_{p+7 n+2}}<x_{p+7 n+3}<\frac{1}{x_{p+7 n+5}} \\
& \text { (iv) } x_{p+7 n+1}<\frac{1}{x_{p+7(n-1)+6}} .
\end{aligned}
$$

Finally, we have

$$
1<x_{p+7(n+1)}<x_{p+7 n+5}<\frac{1}{x_{p+7 n+3}}<x_{p+7 n+1}<\frac{1}{x_{p+7(n-1)+6}}<
$$




$$
<\frac{1}{x_{p+7(n-1)+4}}<x_{p+7(n-1)+2}<x_{p+7(n-1)} .
$$

From this, one see that $\left\{x_{p+7 n}\right\}_{n=0}^{\infty}$ is decreasing with lower bound 1 . So, the limits

$$
\begin{aligned}
L & =\lim _{n \rightarrow \infty} x_{p+7 n}=\lim _{n \rightarrow \infty} x_{p+7 n+5}=\lim _{n \rightarrow \infty} \frac{1}{x_{p+7 n+3}}=\lim _{n \rightarrow \infty} x_{p+7 n+1} \\
& =\lim _{n \rightarrow \infty} \frac{1}{x_{p+7 n+6}}=\lim _{n \rightarrow \infty} \frac{1}{x_{p+7 n+4}}=\lim _{n \rightarrow \infty} x_{p+7 n+2}
\end{aligned}
$$

exist and finite.

Noting that

$$
x_{p+7 n+6}=\frac{x_{p+7 n+5}^{b} x_{p+7 n+4} x_{p+7 n+2}^{b}+x_{p+7 n+5}^{b}+x_{p+7 n+4}+x_{p+7 n+2}^{b}+a}{x_{p+7 n+5}^{b} x_{p+7 n+4}+x_{p+7 n+4} x_{p+7 n+2}^{b}+x_{p+7 n+5}^{b} x_{p+7 n+2}^{b}+1+a}
$$

and taking limit on both sides of this equation, we obtain

$$
\frac{1}{L}=\frac{L^{2 b-1}+2 L^{b}+L^{-1}+a}{2 L^{b-1}+L^{2 b}+1+a} \Longrightarrow L=1 .
$$

Up to now, in the first case we have shown

$$
\lim _{n \rightarrow \infty} x_{p+7 n+k}=1, \quad k=\overline{0,6} .
$$

For the second case

$$
\ldots, 3^{+}, 2^{+}, 1^{-}, 1^{+}, 3^{-}, 2^{+}, 1^{-}, 1^{+}, \ldots
$$

One can prove by the similar way, it is omitted. The proof is complete.

\section{References}

[1] M. Bayram and S. E. Das, Global asymptotic stability of a nonlinear recursive sequence, Int. Math. Forum 5-22 (2010), 1083-1089.

[2] V. L. Kocic, G. Ladas, Global behavior of nonlinear difference equations of higher order with applications, Kluwer Academic, Dordrecht, 1993.

[3] V. V. Khuong, Qualitative properties for a fourth-order rational difference equation (II), Int. J. Math. Analysis, Vol. 4, 2010, No. 13, 617-629.

[4] V. V. Khuong, Qualitative properties for a fourth-order rational difference equation (III), Int. J. Math. Analysis, Vol. 3, 2009, No. 35, 17231733. 
[5] X. Li, D. Zhu, Global asymptotic stability of a nonlinear recursive sequence, Appl. Math. Appl. 17 (2004), 833-838.

[6] X. Li, Qualitative properties for a fourth-order rational difference equation, J. Math. Anal. Appl. 311 (2005), 103-111.

[7] X. Li, Global behavior for a fourth-order rational difference equation, $J$. Math. Anal. Appl. 312 (2005), 555-563.

Received: March, 2011 